\title{
Unternehmerische Verwertung wissenschaftlicher Ergebnisse
}

\section{Empfehlungen für das Konzipieren eines Gründungs-TÜV}

Ein Prototyp für die unternehmerische Verwertung wissenschaftlicher Ergebnisse von Studierenden und Absolventen sind Startups. An ihrem Anfang stehen eine innovative Idee, eine Technologie, ein Geschäftsmodell und in der Regel geringe finanzielle Ressourcen - aber: Sie haben ein großes Wachstums- oder mindestens sehr wissensintensives Potenzial. Gespräche mit zahlreichen Gründern und Mitarbeitern des Gründerservices sowie die einschlägige Literatur verweisen auf den nachhaltigen Einfluss der ersten Schritte für den unternehmerischen Erfolg. Der vorliegende Text möchte diese darstellen und dabei vor allem den Anspruch an die Konzipierung der Gründungsidee mittels Gründungs-TÜV verdeutlichen. Er soll helfen, Verwertungschancen zu beurteilen, Selbstsicherheit zu gewinnen und Promotoren zu überzeugen.

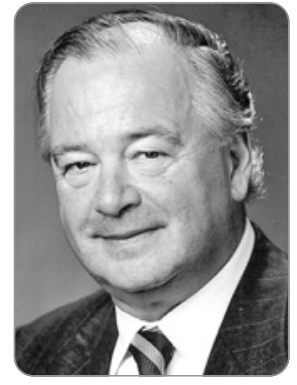

Prof. Dr. Günter Lehmann

ist Vizepräsident des Europäischen Instituts für postgraduale Bildung an der TU Dresden (EIPOS) sowie Dozent an der Dresden International University (DIU). Bevorzugte Forschungsgebiete: Techniken wissenschaftlichen Arbeitens, Ingenieurpädagogik.

Stichwörter: Gründen, Startup, Lean-Startup, Gründungsidee, Gründungsteam

\section{Kennzeichnung}

Der Absolvent einer Universität oder Hochschule - mitunter auch noch Studierender oder bereits Wissenschaftlicher Mitarbeiter - hat sich entschlossen, auf die relative Sicherheit in einem Angestelltenverhältnis zu verzichten und ein Unternehmen - ein Startup - zu gründen. Dazu braucht der Gründer ein Produkt bzw. eine Dienstleistung, Kunden, die bereit sind, dafür zu zahlen und einen Weg, um selbst bezahlt zu werden. Die Idee zur Selbstverwertung in einem eigenen Unternehmen entsteht in der Regel nicht unmit- telbar aus den Ergebnissen der Graduierungsarbeit. Zwar gibt es Fälle, in denen Gründungsideen aus Master- oder Diplomarbeiten entwickelt wurden. Aber viel häufiger entstehen sie auf anderen Pfaden.

Der Weg bis zum vollen Geschäftsbetrieb eines Startups ist in der Abb. 1 dargestellt. Er besteht in der Regel aus drei Etappen. Die erste Etappe, die Entwicklung verläuft vom Aufkeimen der Gründungsidee bis zum Aufbau des Geschäftskonzepts. In der zweiten Etappe erfolgt die Institutionalisierung, vom Erstellen des Businessplanes bis zur Gründung des Startups. Die Umsetzung der im Businessplan manifestierten Gründungsidee, also der Übergang zum vollen Geschäftsbetrieb, füllt die dritte Etappe aus. Alle Etappen, insbesondere das Institutionalisieren und das Umsetzen, werden von der Professionalität der Transferstellen (Gründerservice) begleitet, die heute an vielen Universitäten und Hochschulen Neugründungen unterstützen (vgl. Plum, 2016, S. 12 f.).

\section{Entdeckung}

Erfolgreiche Gründer aus Absolventenkreisen legen die Spuren für die Selbstständigkeit schon in den ersten Studienjahren (vgl. Gschwandtner, 2018, S. 12 ff.). In Praktika 


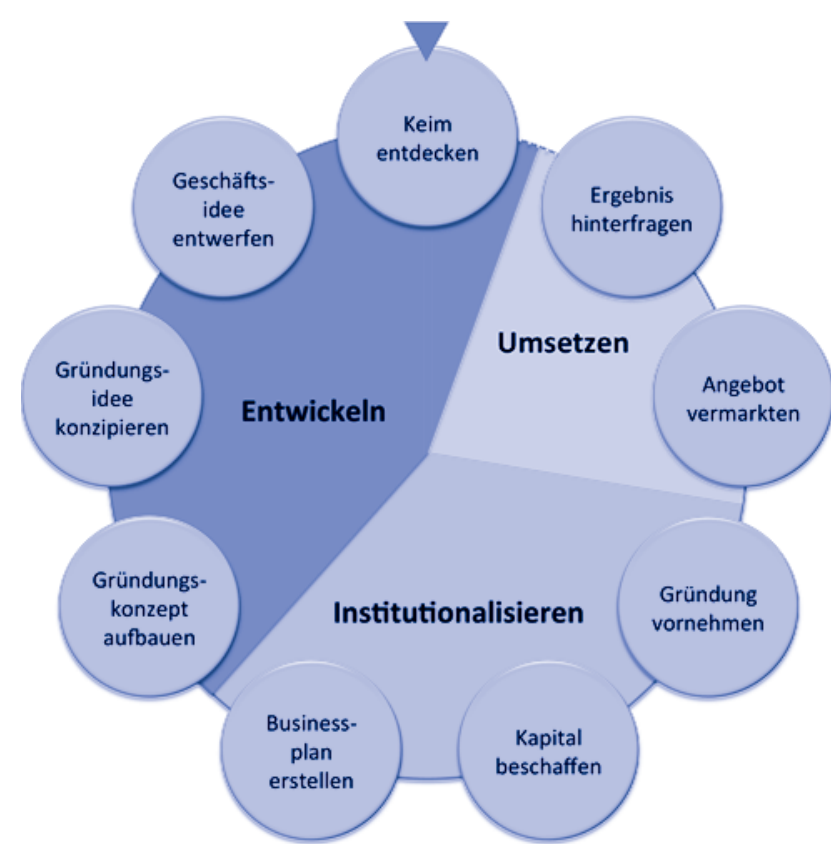

Abb. 1: Etappen und Schritte auf dem Weg zum Geschäftsbetrieb eines Startups

erlebten sie innovative Prozesse. Nebenjobs haben sie nicht nur aus finanziellen Gründen ausgewählt, sondern auch, um sich auf verschiedenen Gebieten auszuprobieren. Dabei wurden Prozesse verstanden, Projektmanagement erlebt und vor allen erfahren, wie Ideen im Hinblick auf ihre ökonomische Verwertbarkeit zu beurteilen sind.

Beispiel:

Bill Gates hatte als Student in einem Praktikum die Idee, ein Betriebssystem für Computer zu entwickeln. Diese innovative Geschäftsidee machte ihn zum reichsten Mann der Welt.

Das Beispiel zeigt: Frühzeitig entdecken spätere Gründer ihr Unternehmer-Gen (Entrepreneur-Gen). Sie streben weniger nach einem Chefposten im DAX-Unternehmen, sondern nach einem hohen $\mathrm{Ma}$ an Selbstständigkeit beim Verwirklichen ihrer interessanten Ideen in einem kleinen Kreis von Mitstreitern. Sie finden heraus, was sie gut können und was ihnen weniger gut gelingt. Sie erfahren, wo sie trotz Krafteinsatz Durchschnitt bleiben (z.B. selber produzieren) und auf welchen Gebieten sie Überdurchschnittliches leisten können (z.B. präsentieren, verkaufen).

Von der Entdeckung ihrer Ambitionen motiviert, besuchen sie besondere Lehrveranstaltungen, z.B. zum Thema "Gründungsorientierte Einführung in die Betriebswirtschaftslehre" oder Veranstaltungen, in denen Gründungsgeschichten von erfolgreichen Unternehmern vorgestellt werden. Sie nehmen Jobangebote als studentische Mitarbeiter und Praktika in einem Gründungsteam an (vgl. Gschwandtner, 2018, S. 65). Beispielhaft sei hier die Hochschulallianz für angewandte Wissenschaften genannt, in der sich sechs deutsche Hochschulen u.a. zur Unterstützung von Startup-
Gründungen zusammengeschlossen haben. Studierende können sich mit ihrer Geschäftsidee bewerben und ein sechstägiges Gründertraining in Berlin gewinnen (www.haw tech.de/startuplab).

In diesem Prozess der aktiven Auseinandersetzung keimt die Geschäftsidee, setzt ihre Entwicklung ein. Sie ist der Ausgangspunkt für eine sehr persönliche Entscheidung, die Gründung des Startups anzugehen. Frühzeitig sollten jetzt die Gesprächsangebote der Transferstellen genutzt werden, um eine erste Einschätzung der Geschäftsidee, Hilfen beim späteren Entwickeln der Gründungsidee und Erstellen des Geschäftskonzepts zu erhalten. Gefragt ist auch die Unterstützung für die Startfinanzierung bis hin zur Beantragung eines Gründerstipendiums. Daneben vernetzen diese Stellen die Gründungswilligen mit Experten nicht nur innerhalb der Wissenschaft, sondern auch mit Experten am Markt. An einigen Universitäten ist die Gründungsberatung als 0pen-Stop-Agency organisiert. Hier kann sich jeder Interessent, unabhängig vom Stand der Ideenentwicklung, Rat holen (vgl. Walter, 2018, S. 95).

\section{Geschäftsidee}

Die Entwicklung der Geschäftsidee verläuft in einer Vorphase der Unternehmensgründung. Sie wird begleitet von einer intensiven Beratung durch die Transferstelle. In dem sich die Konturen der Idee ausschärfen, entstehen auch Vorstellungen über notwendige Geschäftsbereiche. Mit Blick auf die eigenen Stärken beginnen die Recherchen nach Partnern, die bestimmte Aufgaben im Unternehmen noch besser ausfüllen können, als der Urheber selbst.

Aus der Fülle der beim ökonomischen Verwerten von Erkenntnissen gesammelten Erfahrungen, aus dem praktischen Erleben heraus entstehen Geschäftsideen, z.B. durch

- Ableiten aus aktuellen Trends (beispielsweise CouchSurfing oder HelloFresh),

- Verändern der bestehenden Technik,

- neues Design für Produkte,

- Verbessern des Service,

- Optimieren von Prozessen, z.B. in Gesundheitseinrichtungen,

- Erfinden eines gänzlich neuen Produkts oder einer Dienstleistung,

- Verbessern bereits erfolgreicher Geschäftsideen (beispielsweise zusätzliche Funktionen, geringerer Preis oder verbesserte Qualität).

Unter den Produktideen bestimmen vor allem die Qualität und der Absatz neuer Produkte die Erfolgschancen auf dem Markt. Demgegenüber werden neue Dienstleistungen besonders durch die Kompetenz und Flexibilität der handelnden Personen erfolgreich etabliert. Im ständigen Kontakt mit der Transferstelle wird aus der Vielzahl der Überlegun- 
gen die Geschäftsidee verständlich formuliert. Sie enthält Antworten auf folgende Fragen:

- Wer soll das Produkt kaufen bzw. die Dienstleistung annehmen?

- Welche Bedürfnisse hat diese Zielgruppe?

- Was benötigt die Zielgruppe?

- Gibt es vergleichbare Angebote auf dem Markt?

- Wie können die Bedürfnisse der Zielgruppe befriedigt werden?

Eine Geschäftsidee in diesem Stadium sollte in etwa mit fünf Sätzen formuliert und mehreren Personen zum kritischen Lesen gegeben werden. Man bedenke: $0 \mathrm{~b}$ eine Idee gut und richtig ist, erweist sich in den ersten Kundenkontakten. Deshalb werden vielfältige Interaktionen mit der Zielgruppe empfohlen. Befürchtungen, die eigene Idee öffentlich zu machen und damit an andere zu verlieren, sollte nicht nachgegeben werden. Der eigene Entwicklungsvorsprung ist kaum einzuholen, eventuell durch ein Patent abzusichern (vgl. Naumann, 2018, S. 947).

\section{Gründungsidee}

Die Entwicklung der Gründungsidee aus der Geschäftsidee ist ein wichtiger Meilenstein auf dem Wege zum Startup. Jetzt muss der Gründer vor dem kritischen Prüfstein Investor bestehen. Lewis (2019) bezeichnet diese Prüfung als "Gründungs-TÜV". Die erste und entscheidende Frage des Investors lautet: Welcher Gewinn ist von der Umsetzung der Innovation als Produkt aus Menge und Marge zu erwarten?

Die Menge wird durch den Markt, die Marge durch die Industrie bestimmt. Dieser Sachverhalt wird in $A b b .2$ mit der
Kennzeichnung der zwölf Elemente des Gründungs-TÜV vorgestellt.

Auf den ersten Blick lassen sich aus Abb. 2 fünf Fragen für das Verwerten der Innovation ableiten:

- Wie attraktiv ist der Markt?

- Wie attraktiv ist die entsprechende Industrie?

- Welche Kunden werden angesprochen (Segmentierung)?

- Welche Wettbewerbsvorteile werden erwartet (Preis/Kosten)?

- Welches Team sichert den Erfolg?

Auf den zweiten Blick wird deutlich, dass jede dieser Fragen mit präzisen Nachweisen zu beantworten ist. Auf der Makroebene sind für die Attraktivität des Markts (z.B. Kleidung) zwei Fragen zu beantworten:

(1) Wie groß ist der Markt?

(2) Wie wird der Markt in den nächsten Jahren wachsen?

In der Mikroebene ist zu fragen:

(3) In welchem Teil des Markts (Kinderkleidung) ist die Innovation angesiedelt? Welches Segment wird angezielt (z.B. Altersgruppen)?

Die Attraktivität der Industrie (z.B. Textil- oder Chemische Industrie), die für die Verwertung der Innovation von Bedeutung ist, liefern Antworten auf die folgenden Fragen:

(4) Welche Konkurrenzsituation besteht, z.B. Oligopol?

(5) Wie werden Quantität und Qualität der Lieferanten eingeschätzt?

6) Mit welchem Kundenbestand ist zu rechnen (Konsumenten, Händler, Berater etc.)?

(7) Gibt es Neueinsteiger in dasselbe oder ein ähnliches Geschäft?

(8) Welche Substitute existieren, mit welchen Ersatzprodukten werden die gleichen Bedürfnisse befriedigt?

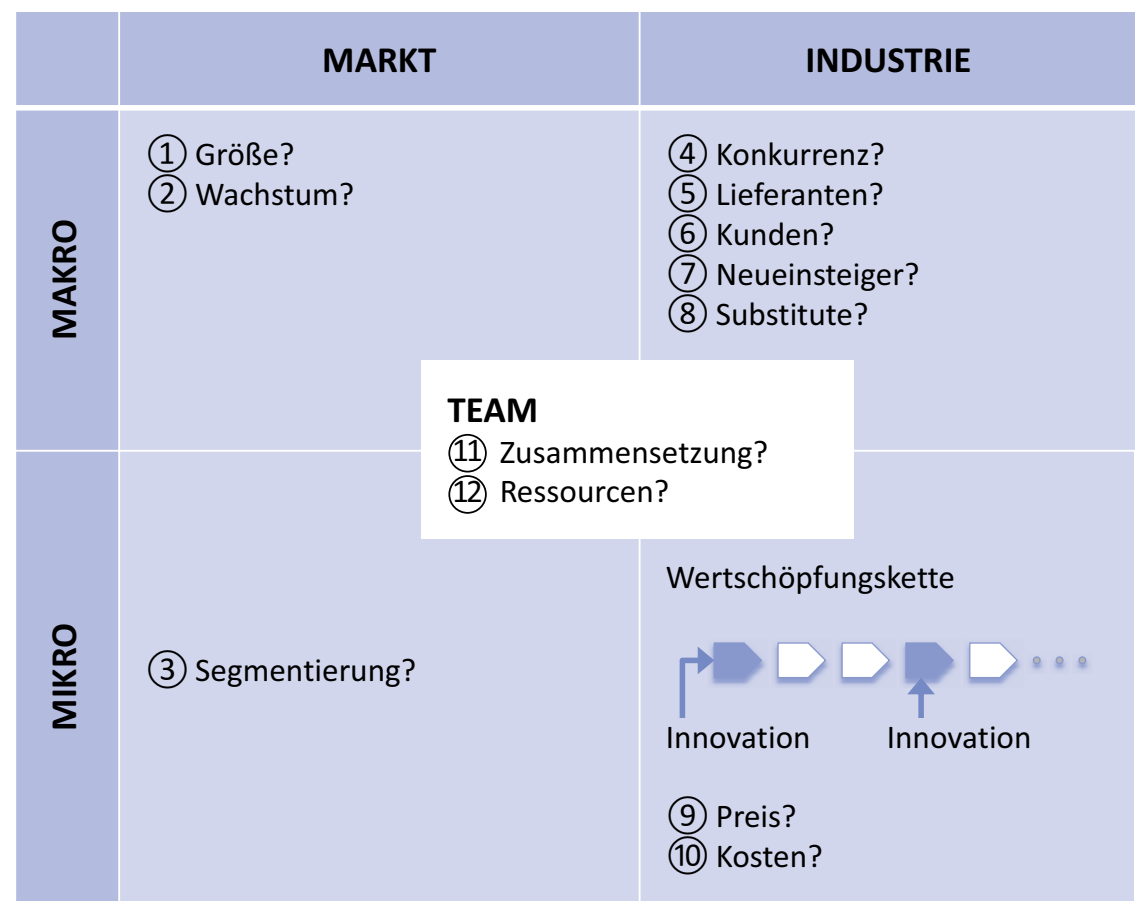

Quelle: In Anlehnung an Lewis (2019)

Abb. 2: Gründungs-TÜV 
Aus den Antworten auf die Fragen (5) bis (8) kann in erster Näherung das Gewinnpotential der Innovation abgelesen werden.

Auf der Mikroebene ist der erwartbare Gewinn zu ermitteln. Dazu sind folgende Fragen zu beantworten:

(9) Welcher Preis soll erzielt werden (kann durch Innovation positiv beeinflusst werden)?

(10) Welche Kosten müssen aufgewendet werden?

Bei der Gewinnermittlung steht die Innovation nicht nur am Anfang der Wertschöpfungskette. So kann beispielsweise am gleichen Produkt durch Innovationen in der Fertigung oder im Vertrieb eine Kostensenkung und damit eine Erhöhung des Gewinns erzielt werden (siehe Abb. 2).

Alle bisher beantworteten Fragen geben Aufschluss über die Chancen der Gründungsidee. Aber Gewissheit entsteht erst, wenn das Gründungsteam transparent wird. Dazu sind vor allem die folgenden zwei Fragen zu beantworten:

(11) Wie ist das Team zusammengesetzt, wie viel und welche Personen gehören mit welcher Qualifikation da$\mathrm{zu}$ ?

(12) Über welche Fähigkeiten und welche Ausrüstungen, Mittel, Materialien etc. verfügt das Team?

Die Gründungsidee ist erst dann reif, wenn alle zwölf Fragen im Gründungs-TÜV beantwortet sind. Damit liegt ein gründlich durchdachtes Werkzeug vor, um Investoren zu gewinnen und gemeinsam mit den Beratern aus der Transferstelle das Gründungskonzept, den Gründungsgeschäftsplan zu erstellen.

Bei Gründungen aus dem Studium heraus entsteht mitunter das Problem, dass der Gründungswillige für seine Gründungsidee schon einen Investor gewonnen hat und jetzt starten möchte, aber die Diplom- oder Masterarbeit steht noch aus. Was ist die Folge? Soll die Gründung vollzogen und die Exmatrikulation wegen verspäteter Abgabe der Graduierungsarbeit in Kauf genommen werden? Oder soll auf die Gründung und damit auch auf den Investor verzichtet und die Arbeit pünktlich abgeliefert werden? Die Transferstelle findet sicher mit einer gründungsfreundlichen Universitätsverwaltung eine Regelung, um beiden Anliegen gerecht zu werden (vgl. Walter, 2018, S. 957).

\section{Geschäftskonzept}

Das Geschäftskonzept soll eine gründliche Planung des Gründungsprozesses und der zukünftigen Abläufe ermöglichen und ist im Businessplan festzuhalten.

Das Dokument enthält in Anlehnung an Plum/Gehrer/ Schmidt (2016, S. 66) in der Regel:

- Die Geschäftsidee (zusammenfassende Darstellung von Kapitel 4),

- Beschreibung der Geschäftstätigkeit (Dienstleistungsbzw. Produktkonzept),
- Die Marktanalyse (Kundenprofile, Konkurrenzanalyse, Standortanalyse, Gesamtanalyse des Marktes).

- Das Zukunftskonzept (Vision, endgültiges Produkt- und Servicekonzept, endgültige Zielgruppendefinition, Strategie, Erfolgsfaktoren, Management, Organisation, Standort).

- Das Konzept zu Recht, Steuern und Versicherungen (Rechtsform, Steuerplanung, erforderliche Versicherungen).

- Der Finanzplan (Investitionen, Aufwendungen, Umsatzplan, Finanzierungsplan, Liquiditätsrechnung).

- Das Marketingkonzept (zielgruppenbezogene Marketingkonzepte mit der Definition der Produkt-, Service-, Preis-, Distributions- und Kommunikationspolitik).

- Die Gründerperson(en) und das Personal.

- Das Geschäftsprofil (das Unternehmenskonzept) wird auf einer Seite zusammenfassend mit dem Firmennamen und -sitz dargestellt.

- Anlagen (Projektplan mit Meilensteinen, Gesellschafterertrag, technische Gutachten, Patente etc.).

Damit liegt ein ausgereifter Plan vor, mit dem der Gründer auf den Markt gehen kann.

\section{Gründungsteam}

Die Formierung des Gründungsteams vollzieht sich über die gesamte Entwicklungsphase.

Der Rat eines erfahrenen Startup-Gründers lautet: Ich setze mein Team nicht aus Freunden zusammen, sondern suche mir die Besten, die ich gewinnen kann. Sein Ziel ist dabei, das Gründungsteam durchweg mit überdurchschnittlichen Leistungsträgern zu besetzen. Wenn der Ideengeber selbst ein sehr guter Programmentwickler ist, sollte er die Leistungsfähigkeit des Teams beispielsweise durch exzellente Planer und Verkäufer ergänzen. Wer ist für welche Aufgabe besonders geeignet, welche Rolle spielt der Ideengeber, ist ein anderer, mit unternehmerischer visionärer Persönlichkeit, für die Leitung des Teams vielleicht effektiver? Persönliche Befindlichkeiten und Eitelkeiten sind hinderlich.

Typische Rollen im Startup-Team sind

- Der Entwickler - entwickelt die Technologie zur Umsetzung der Idee mit besonderen Fähigkeiten zum Problemlösen u.a. im IT-Bereich.

- Der Designer - treibt alle Prozesse im Unternehmen an, ist Kreativgeist, hat Visionen zur Marktentwicklung.

- Der Marketer - verkauft das Ergebnis, spricht die Zielgruppen an, sichert Kundenzufriedenheit, verbreitet positive Stimmung nach innen und außen.

- Der Kaufmann - sichert die Wirtschaftlichkeit, lotet Chancen und Risiken am Markt aus und ist für den Kapitalgeber Garant für die effiziente Verwendung seiner Einlage. Außerdem ist er unverzichtbar bei Bewerbungen und Antragstellungen. 
Unternehmensgründer finden ihre Partner häufig in gemeinsamen Aktionen, beispielsweise in Nebenjobs, Projekten, Workshops und den zahlreichen Startup-Veranstaltungen der Transferstellen. Für besondere Aufgaben im Team sind Ausschreibungen üblich - sowohl national als auch zunehmend international. In der Regel bestehen die Teams aus zwei bis sechs Personen, ergänzt durch studentische Hilfskräfte und Praktikanten.

Eine wesentliche Bedingung für die Arbeit im Team ist, dass sich alle voll mit der Unternehmensidee identifizieren. Die Partner sind nicht schlechthin Mitarbeiter, sondern Mitunternehmer. Jeder muss seine Rolle kennen und aktiv vertreten. In einer offenen Gesprächskultur werden Unstimmigkeiten offen thematisiert.

In dieser Gemeinschaft wird das Konzept für die Gründung des Unternehmens erarbeitet. Für die zahlreichen Abläufe bei der Entwicklung von der Geschäftsidee zur Gründungsidee und dem Geschäftskonzept wird auf die Vorschläge für moderierte Gruppendiskussion und Ergebnispräsentation in Lehmann, 2020, S. 53 ff. verwiesen.

\section{Quintessenz}

Folgt man den Berichten von Startup-Gründern und Mitarbeitern von Transferstellen, so lässt sich festhalten:

1. Startups sind nicht das "Produkt” einer konzertierten Aktion der Universität oder Hochschule. Studenten entdecken ihr Gründer-Gen im eigenen Erleben, in der gestaltenden Aktion, in der Mitwirkung an herausfordernden Projekten und sicher auch in inspirierenden Lehrveranstaltungen und Gründerforen. In der weitestgehend selbstgesteuerten Wegführung entsteht ihre unternehmerische Intension, entdecken sie ihre besonderen Fähigkeiten (und sicher auch Grenzen) und setzen sie zielorientiert ein.

2. Die Universitäten und Hochschulen schaffen dafür ein Umfeld, das offen und fördernd für unternehmerisches Denken und Handeln ist. Dafür bieten sie Lehrveranstaltungen zum Erwerb von betriebswirtschaftlichen und Rechtskenntnissen, vermitteln in Veranstaltungen Erfahrungen junger und erfahrener Gründer, bieten Praktika, Nebenjobs, studentische Mitarbeit in Forschungsund Entwicklungsprojekten an.

3. In diesem aktionsreichen Feld können die künftigen Gründer ihre Ideen entwickeln und prüfen. Sie entdekken ihre Stärken, aber auch die Mittelmäßigkeit bestimmter eigener Fähigkeiten und stellen dabei fest, was zu ergänzen ist und in welcher Partnerschaft sie die Geschäftsidee optimal umsetzen können.
4. Spätestens beim Stand dieser Erkenntnis, besser noch früher, springen die Transferstellen innerhalb der Universitäten und Hochschulen oder in ihrer unmittelbaren Nähe beratend ein. Sie sind mit ihren Erfahrungen ein kritischer Prüfstein für die Geschäftsidee, später ein unverzichtbarer Partner bei der Prüfung der Geschäftsidee (Gründungs-TÜV) und ihrer Überführung in den Businessplan.

5. Eine gründerfreundliche Universitäts- bzw. Hochschulverwaltung schafft gemeinsam mit der Transferstelle Regelungen, dass der gründungswillige Student sein Startup rechtzeitig installieren und zugleich sein Studium ordnungsgemäß mit der Graduierungsarbeit beenden kann.

\section{Literatur}

Barbarski, K., Lean Startup Methode: So entwickelt Ihr schlank und schnell Geschäftsmodelle. https://www.gruenderkueche.de/lexikon/begr iffe/lean-startup-so-entwickelt-ihr... (Abrufdatum: 24.07.2019).

Bessan, H./Krais, P./Wittstock M., Machen. Der Startup - Buch des mymuesli-Gründers, Hamburg 2017.

Deutscher Hochschulverband, Start-Up Kolumne, in: Forschung \& Lehre, 25. Jg. (2018), S. 940-965.

Gschwandtner, F., Mein Leben, meine Erfolgsgeheimnisse, Salzburg/München 2018.

Guillebeau, C., Start-up! Wie Sie mit weniger als 100 Euro ein Unternehmen auf die Beine stellen und Ihr eigener Chef werden, 4.Aufl., Kulmbach 2018.

HAWtech, Startup-Lab, HochschulAllianz für Angewandte Wissenschaften, Hochschulen aus Berlin, Darmstadt, Dresden, Esslingen, Karlsruhe und Aachen. https://www.hawtech.de/startuplab. (Abrufdatum: 25.07.209). Lehmann, G., Die Arbeit ist fertig - was nun? Empfehlungen zum Umsetzen von Ergebnissen aus Graduierungsarbeiten, in: WiSt-Wirtschaftswissenschaftliches Studium, 49. Jg. (2020), Nr. 4, S. 53-58.

Lewis, G., Ein TÜV für die Startup-Gründungsidee. Gespräch mit Prof. Dr. G. Levis, Hochschule für Technik und Wirtschaft, Fakultät für Wirtschaftswissenschaften, am 14.02.2019.

Maurya, A., Scaling Lean. Wie Startups wachsen, München 2019.

Naumann, T., Das Unternehmertum kommt an der Universität zu kurz. Interview in der Rubrik START-UPS, in: Forschung \& Lehre, 25. Jg. (2018), S. $947-948$.

Ossola-Haring, C./Dürr, A. Erfolgreich gründen. Start-Up im Studium, Tübingen 2016.

Plum, B./Gehrer, M./Schmidt, J., Existenzgründung für Hochschulabsolventen. Geschäftsidee, Business Plan, Fördermittel, Kundenakquise, Crowdfunding, Freiburg/München/Stuttgart 2016.

Renk, E., Das Feierabend Startup. Risikolos gründen neben dem Job, 2. Aufl., München 2018.

Schulte, R., Ideennester, Start-ups und ihre Bedingungen, Interview, in: Forschung \& Lehre, 25. Jg. (2018), S. 942-943.

Thönnessen, F., Arbeitsbuch Start-Up. Das 7-Stufen-Programm, München 2018.

Walter, A., S., 2018. Hausaufgaben der Hochschule. Gründungsförderung in der Wissenschaft, in: Forschung \& Lehre, 25. Jg. (2018), S. 956. 